\title{
Prime and pull against HSV-2
}

\section{By Michael J. Haas, Senior Writer}

Yale School of Medicine researchers have mouse data showing that vaginal application of two chemokines recruits memory $\mathrm{T}$ cells into vaginal tissue and improves the protective effect of an HSV-2 vaccine. ${ }^{1}$ The team is now working to prolong the duration of the chemokines' effects and wants to extend the strategy to HIV prophylaxis.

Memory T cells circulate freely throughout many organs of the body but cannot enter vaginal mucosae-and certain other tissues such as skin and lung airways - until recruited by chemokines in response to inflammation or infection. This mechanism restricts the ability of memory $\mathrm{T}$ cells induced by vaccines to reach and protect vaginal tissues prior to viral infection, thus blunting efficacy.

"We hope to extend the strategy to males in the future because - in principle-topical application of chemokines could establish memory $T$ cells in the male genital tract."

-Akiko Iwasaki, Yale School of Medicine
In 2009, a group of Yale researchers led by Akiko Iwasaki uncovered a potential way around the tissue entry roadblock. They showed in mice that vaginal infection with herpes simplex virus-2 (HSV-2) induced local expression of two chemokines-chemokine CXC motif ligand 9 (Cxcl9; Mig) and Cxcl10 (Ip-10). Those two chemokines recruited $\mathrm{T}$ cells expressing CXC chemokine receptor $3(\mathrm{Cxcr} 3)$ into vaginal tissue. ${ }^{2}$

These findings led the researchers to hypothesize that they could improve the prophylactic effects of an HSV-2 vaccine by harnessing signaling between CXCL9 or CXCL10 and CXCR3.

In the new study, the group first looked at whether CXCL9 and CXCL10 could recruit virus-specific $T$ cells to the vagina in the absence of infection. The team injected mice with HSV-2-specific human $\mathrm{CD}^{+} \mathrm{T}$ cells and inoculated the animals with a subcutaneous HSV-2 vaccine to induce expansion of those T cells.

Indeed, topical application of CXCL9 and CXCL10 to the vaginal tract increased levels of the virus-specific T cells compared with no chemokine treatment. The effect lasted for at least 12 weeks.

Next, the team investigated whether inoculation with an HSV-2 vaccine followed by topical application of the two chemokines to the vaginal tract-a strategy they dubbed prime and pull-would protect mice from HSV-2 infection. Mice receiving lethal vaginal challenge with the virus at
4 or 12 weeks after prime and pull had fewer symptoms of infection, lower viral titers in peripheral dorsal root ganglia and longer survival than mice given the vaccine alone.

Lastly, the team showed that topical application of CXCL9 and CXCL10 to the vaginal tract did not recruit other immune cells to the tissue or induce a local or systemic inflammatory response.

Iwasaki is professor of immunology and of molecular, cellular and developmental biology at the Yale School of Medicine. She conducted the study with Haina Shin, a postdoctoral fellow in her immunology research group.

Data were reported in Nature.

"The concept of giving chemokines or cytokines to develop and strengthen the immune response to vaccines is not new, but systemic administration of the molecules leads to unacceptable side effects such as pain, inflammation and cell damage," said Gregory Stoloff, founder and CEO of Seek Ltd. "The Yale researchers have taken a different route by applying the chemokines topically_thereby avoiding or reducing side effects-and showing that the molecules can penetrate the skin and vaginal wall to call T cells to the site."

He added that the study "reinforces the idea that stimulating $\mathrm{T}$ cells and getting them into vaginal tissues before infection is possible and has a greater protective effect" than vaccination alone.

Seek's HIV-v, a T and B cell vaccine targeting the Nef, Rev, Vif and Vpr noncapsid HIV proteins, has completed Phase Ib/II testing to treat HIV.

Jessica Baker Flechtner, VP of research at Genocea Biosciences Inc., agreed. "This is an innovative strategy to efficiently target the right cells to the right place at the right time," she said.

Genocea's GEN-003, a vaccine consisting of the infected cell polypeptide 4 (ICP4) and gD2 antigens from HSV-2 combined with the saponinderivative adjuvant Matrix M, is in Phase I/Ila testing to treat patients with moderate to severe HSV-2 infection who are otherwise healthy.

Past strategies aimed at achieving the same objective included intravaginal-rather than intramuscular-vaccination and applying a chemical pull signal to the genital mucosa without an initial prime vaccination. "Neither approach appears to have been as successful as that proposed by these authors," she said.

As examples, she cited imiquimod and the related compound resiquimod, "which likely have no direct antiviral effect but instead promote antigen-specific T helper type 1 immunity when administered topically."

Meda AB and Medicis Pharmaceutical Corp. market Aldara imiquimod, a 5\% imiquimod cream that agonizes toll-like receptor 7 (TLR7), to treat genital warts, actinic keratosis and basal cell carcinoma (BCC).

In 2003, 3M Co. and Eli Lilly and Co. suspended three Phase III trials of resiquimod-an immune response modifier that agonizes TLR7 and TLR8-to treat genital herpes after preliminary data showed that the doses used would not achieve adequate efficacy.

\section{Pushing limits}

Both Flechtner and Stoloff said the prime and pull strategy faces several logistical obstacles that could limit its clinical application. 
For example, Flechtner said the two-part immunization regimen would require two clinic visits within a precise window of time to be effective and thus could run into compliance issues.

Both she and Stoloff said specialized training might be needed to ensure proper administration of the chemokine pull. The team "might benefit from discussing the strategy with primary care physicians to determine how something like this may be implemented in practice," Flechtner said.

"Another problem is that the T cells won't remain in the restricted tissues for more than a few months," Stoloff told SciBX. "So you couldn't apply a topical formulation of the chemokines and expect the effects to last four or five years. To keep the memory $\mathrm{T}$ cells in the vaginal tissues, you would have to apply the chemokines every six months or so, and unfortunately this is not practical."

Lisa Wei, head of HSV research at GenVec Inc., agreed. The team's study "demonstrates the potential for a single vaccination with a chemokine pull to confer about three months of protection," but further studies will have to determine whether prime and pull can boost vaginal mucosal immunity in humans and if so, for how long, she said.

GenVec's HSV-2, a vaccine to prevent and treat HSV-2 infection, is in preclinical testing.

Iwasaki acknowledged that it was not clear yet how long human vaginal tissues would retain $\mathrm{T}$ cells recruited by the chemokines. "But even if we assume long-term $\mathrm{T}$ cell residency is not sustained in humans, we have ways to deliver chemokines locally over a long period," such as the vaginal rings that are already being used to deliver contraceptives, she said.

Iwasaki also said her group is developing techniques to help retain memory $\mathrm{T}$ cells in vaginal tissue for a very long time "or perhaps even for the lifetime of the animal or human." Details on the techniques are undisclosed.

The team also is testing the prime and pull strategy to prevent HIV infection in nonhuman primates.

"We also wish to understand whether prime and pull can be used to treat reactivation of HSV-2" and thus prevent recurrence of the disease in the genital tissues, she said. "We hope to extend the strategy to males in the future because-in principle-topical application of chemokines could establish memory $\mathrm{T}$ cells in the male genital tract."

Yale University has filed a provisional patent covering the findings, and the IP is available for licensing.

Haas, M.J. SciBX 5(43); doi:10.1038/scibx.2012.1130

Published online Nov. 1, 2012

\section{REFERENCES}

1. Shin, H. \& Iwasaki, A. Nature; published online Oct. 17, 2012; doi:10.1038/nature11522

Contact: Akiko Iwasaki, Yale School of Medicine, New Haven, Conn.

2. Nakanishi, Y. et al. Nature 462, 510-513 (2009)

\section{COMPANIES AND INSTITUTIONS MENTIONED}

3M Co. (NYSE:MMM), St. Paul, Minn.

Eli Lilly and Co. (NYSE:LLY), Indianapolis, Ind.

Genocea Biosciences Inc., Cambridge, Mass.

GenVec Inc. (NASDAQ:GNVC), Gaithersburg, Md.

Meda AB (SSE:MEDAA), Solna, Sweden

Medicis Pharmaceutical Corp. (NYSE:MRX), Scottsdale, Ariz.

Seek Ltd., London, U.K.

Yale School of Medicine, New Haven, Conn.

Yale University, New Haven, Conn. 